\title{
Postural Research and Rehabilitation in an Immersive Virtual Environment
}

\author{
E.A. Keshner ${ }^{1,2}$, R.V. Kenyon ${ }^{3}$, Y. Dhaher ${ }^{1,2}$ \\ ${ }^{1}$ Sensory Motor Performance Program, Rehabilitation Institute of Chicago, IL, USA \\ ${ }^{2}$ Dept. of Physical Medicine and Rehabilitation, Feinberg School of Medicine, Northwestern University, IL, USA \\ ${ }^{3}$ Dept. of Computer Science, University of Illinois at Chicago, IL, USA
}

\begin{abstract}
We have united an immersive dynamic virtual environment with motion of a posture platform to record biomechanical and physiological responses to combined visual, vestibular, and proprioceptive inputs. A 6 degree-of-freedom force plate provides measurements of moments exerted on the base of support. Kinematic data from the head, trunk, and lower limb is collected using 3-D video motion analysis. The virtual image is projected via a stereo-capable projector mounted behind the back-projection screen. This system allows us to explore complex behaviors necessary for rehabilitation. We are currently examining how a dynamic visual field affects posture and spatial orientation, and whether visual task demands interfere with our ability to react to a loss of balance in healthy adults and in adults with labyrinthine deficit. Our data suggest that when there is a confluence of meaningful inputs, none of the inputs are suppressed in healthy adults; the postural response is modulated by all existing sensory signals in a non-additive fashion. Labyrinthine deficient adults suppress visual inputs. Individual perception of the sensory structure also appears to be a significant component of the postural response in these protocols. We will discuss the implications of these results for the design of clinical interventions for balance disorders.
\end{abstract}

Keywords - virtual reality, posture, vision, multisensory weighting, labyrinthine deficit

\section{INTRODUCTION}

Models developed from studies of postural behavior in the presence of multisensory inputs have predicted system non-linearities $[6,18]$ but these models have continued to reduce the system to a single degree of freedom or to a single behavioral outcome. Data from our laboratory have strongly indicated, however, that multiple sensory inputs do not demonstrate superposition and that each combination of inputs is differentially weighted across body segments $[11,12]$. Response variability needs to be better characterized before a robust model of postural control can be developed. We are now examining the effect of frequency and magnitude of multisensory inputs to determine the relative weighting of physical and visual stimuli on the postural responses. We employed two longterm sinusoids so that predictive mechanisms were engaged in the reweighting process. Using a virtual environment, visual input was augmented thereby keeping all pathways operational but not equivalent. Relative motion of three body segments was examined to determine whether the

This work was supported by National Institute of Health grants DC01125 from the NIDCD and AG16359 from the NIA. presence of magnitude conflicts between visual and vestibular/somatosensory signals would result in differential weighting.

\section{METHODOLOGY}

A linear accelerator (sled) that could be translated in the anterior-posterior direction was controlled by D/A outputs from an on-line PC. The sled was placed $40 \mathrm{~cm}$ in front of a screen on which a virtual image was projected via a stereocapable projector (Electrohome Marquis 8500) mounted behind the back-projection screen.

The wall in our system consisted of back projection material measuring $1.2 \mathrm{~m}$ x $1.6 \mathrm{~m}$. An Electrohome Marquis 8500 projector throws a full-color stereo workstation field (1024x768 stereo) at $96 \mathrm{~Hz}$ onto the screen. An SGI desk side Onyx II with a Reality Engine created the imagery projected onto the wall. The field sequential stereo images generated by the Onyx II were separated into right and left eye images using liquid crystal stereo shutter glasses worn by the subject (Crystal Eyes, StereoGraphics Inc.). The shutter glasses limited the subject's horizontal FOV to $100^{\circ}$ of binocular vision and $55^{\circ}$ for the vertical direction. The correct perspective and stereo projections for the scene were computed using values for the current orientation of the head supplied by a position sensor (Flock of Birds, Ascension Inc.) attached to the stereo shutter glasses (head). Consequently, virtual objects retained their true perspective and position in space regardless of the subjects' movement. The total display system latency from the time a subject moved to the time the new stereo image was displayed in the environment was 50-75 ms. The stereo update rate of the scene (how quickly a new image is generated by the graphics computer in the frame buffer) was 14-24 stereo frames/sec. Flock of birds data was sampled at $48 \mathrm{~Hz}$.

Scene Characteristics. The scene consisted of a room containing round columns with patterned rugs and painted ceiling. The columns were $6.1 \mathrm{~m}$ apart and rose $6.1 \mathrm{~m}$ off the floor to the ceiling. The rug patterns were texture mapped on the floor and consisted of 10 different patterns. The interior of the room measured $30.5 \mathrm{~m}$ wide by $6.1 \mathrm{~m}$ high by $30.5 \mathrm{~m}$ deep. The subject was placed in the center of the room between two rows of columns. Since the sled was $64.8 \mathrm{~cm}$ above the laboratory floor the image of the virtual room was adjusted so that its height matched the sled height (i.e., the virtual floor and the top of the sled were coincident). Beyond the virtual room was a landscape consisting of mountains, meadows, sky and clouds. The floor was the distance from the subject's eyes to the virtual 


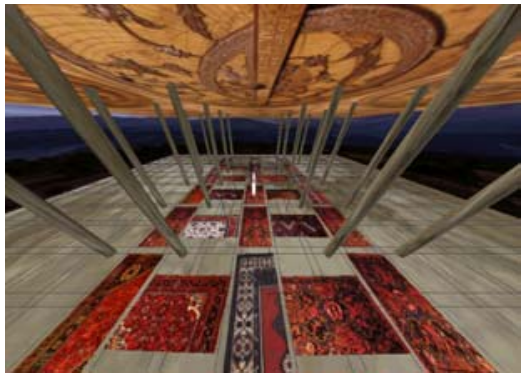

Fig. 1. A birds-eye view of the entire image. The white circle in the center illustrates where the subject is positioned in the image. Only the portion of the image that is in front of the subject can be seen. floor and the nearest column was $4.6 \mathrm{~m}$ away. The resolution of the image was $7.4 \mathrm{~min}$ of arc per pixel when the subject was $40 \mathrm{~cm}$ from the screen. The view from the subjects' position was that objects in the room were both in front of and behind the screen (Fig. 1).

When the scene moved in fore-aft, objects moved in and out of view depending on their position in the scene.

\section{A. Procedures}

Subjects stood on the sled with their hands crossed over their chest and their feet together. Seven healthy young adults (aged 25-38 yrs) and 4 labyrinthine deficient (LD) adults (aged 44-78 yrs) stood on the force platform (sled) in front of a screen on which a virtual image was projected. Either the support surface translated $\pm 15.7 \mathrm{~cm} / \mathrm{sec}( \pm 10 \mathrm{~cm}$ displacement) in the a-p direction at $0.25 \mathrm{~Hz}$, or the scene moved $\pm 3.8 \mathrm{~m} / \mathrm{sec}( \pm 6.1 \mathrm{~m}$ displacement $)$ fore-aft at $0.1 \mathrm{~Hz}$, or both were translated at the same time for $205 \mathrm{sec}$. Trials were randomized for order. In all trials, $20 \mathrm{sec}$ of data was collected before scene or sled motion began (preperturbation period). When only the sled was translated, the visual scene was visible but stationary, thus providing appropriate visual feedback equivalent to a stationary environment.

The sled frequency was selected in order to produce a postural disturbance having minimized intersegmental joint motion [2] but enough vestibular information to be reliable [4]. The visual frequency was selected because Lestienne et al. [14] have demonstrated that the destabilization induced by visual cues conflicting with vestibular and proprioceptive cues is roughly equivalent to the absence of visual cues at frequencies below $0.2 \mathrm{~Hz}$ and postural sway is increased.

\section{B. Data Collection and Analysis}

Three-dimensional kinematic data from the head, trunk, and lower limb were collected at $120 \mathrm{~Hz}$ using video motion analysis (Optotrak, Northern Digital Inc., Ontario, Canada). Infrared markers placed near the lower border of the left eye socket and the external auditory meatus of the ear (corresponding to the relative axis of rotation between the head and the upper part of the cervical spine) were used to define the Frankfort plane and to calculate head position [19]. Other markers were placed on the back of the neck at the level of $\mathrm{C}_{7}$, the left greater trocanter, the left lateral femoral condyle, the left lateral malleolus, and on the translated surface. Markers placed at $\mathrm{C}_{7}$ and the greater trocanter were used to calculate trunk position, and shank position was the calculated from the markers on the lateral femoral condyle and the lateral malleolus.

For trials where the sled moved, sled motion was subtracted from the linear motion of each segment prior to calculating segmental motion. Motion of the three segments was presented as relative segmental angles where motion of the trunk was removed from motion of the head to determine the motion of the head with respect to the trunk. Motion of the shank was removed from motion of the trunk to reveal motion of the trunk with respect to the shank. Motion of the shank was calculated with respect to the sled.

To examine the effect of each protocol on the magnitude of the response across trials, root mean square (RMS) values were calculated over non-overlapping $40 \mathrm{sec}$ intervals of the relative segmental angle data. Power of the response at each stimulus frequency was calculated using a $40 \mathrm{sec}$ sliding window following a fast Fourier transform analysis and plotted across the period of the trial. Data were tested for significance with a repeated measures ANOVA and Bonferroni post-hoc comparisons were performed on the dependent measures at the $p<0.05$ level.

\section{RESULTS}
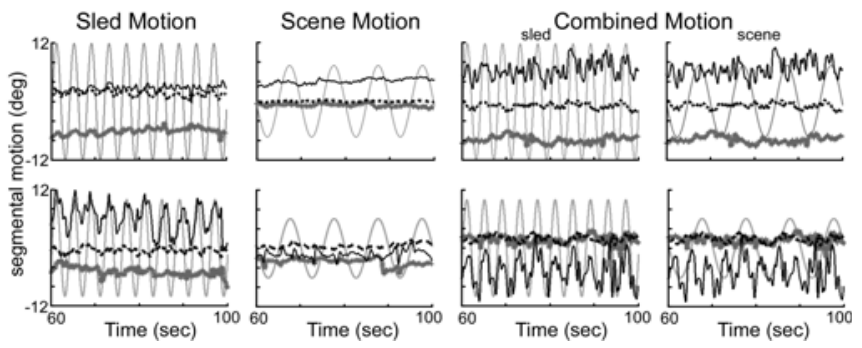

Fig. 2. Relative angles of the head to trunk (bold grey line), trunk to shank (thin black line) and shank to sled (broken black line) are plotted for a $40 \mathrm{sec}$ period of the trial from a healthy adult (top row) and a LD adult (bottom row) during sled motion only, scene motion only, and combined sled and scene motion (the same data are plotted against both the sled and the scene).

\section{A. Responses in Healthy Adults}

When only the sled was moving, there was no conflict between the movement of the sled and visual motion resulting from the body sway. Segmental output was consistent across the whole period of the trial (top row Fig. 2) and response frequencies peaked at the frequency of stimulus input (Fig. 3). Two response strategies were observed. Subjects either compensated for the motion of the sled so that the direction of segmental motion was counter to that of the sled. Or, subjects moved in concert with the sled so that they rode the sled with all segments moving in the same direction. 


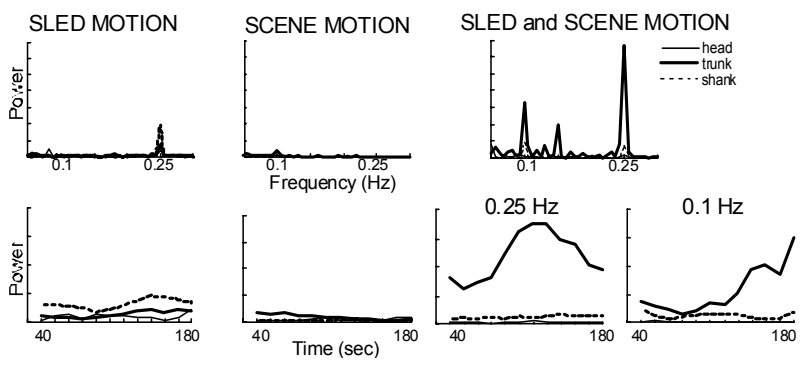

Fig. 3. Power plots of head, trunk, and shank in one healthy adult plotted across frequency (top row) and over the period of the trial (bottom row) at the frequencies of sled motion only $(0.25 \mathrm{~Hz})$, scene motion only $(0.1 \mathrm{~Hz})$ and combined sled and scene motion.

Responses are normalized to the largest response of each segment.

When only the scene was moving, we would expect a conflict between the visual perception of motion and the vestibular and somatosensory systems signaling an absence of physical motion [5]. There was little power at the frequency of the scene in any of the segments (Fig. 3). Small responses of the trunk emerged (Fig. 2) and little relative motion of the head suggested that the head and trunk moved together (Fig. 2). Responses were absent at the shank.

When both the sled and visual scene were moving, the vestibular and somatosensory systems were signaling real motion of the body, but there was a conflict between the visual and the other sensory systems because of the spatial and temporal discordance in the two stimuli. The presence of one frequency for the sled and a different frequency for the visual scene produced an increase in the amplitudes of segmental responses, mostly in the trunk and head (Fig. 2). No habituation was observed over the period of the trial. Increased power that occurred at the stimulus frequencies could not be obtained simply by summing the effects of the trials with single frequency inputs. Power was observed to increase around 60-80 sec into the trial (Fig. 3). A delayed increase in amplitude with increased coherence is similar to a response that we may expect if a subject was experiencing a sensation of self-motion through the visual system known as vection [6,9]. With vection, the optical flow pattern creates a compelling illusion of self-motion that is not corroborated by the inertial forces transmitted through the vestibular sense organs [9]. In our data, the subjects' responses were labile in that they varied in the time of their appearance and could increase and decrease during the course of the trial, which may be related to the subjects' fixation on the visual scene [7].

RMS values of the trunk with combined inputs were significantly greater across all subjects than when standing quietly in the dark and to either the sled or scene motion alone $(F(6,60)=21.15, p<0.0001)$. The same was true of the shank $(F(6,60)=12.47, p<0.0001)$. Thus the effect of discordant stimuli was to significantly increase both the RMS and the power of the segmental response in a nonadditive fashion.

\section{B. Responses in Labyrinthine Deficient Subjects}

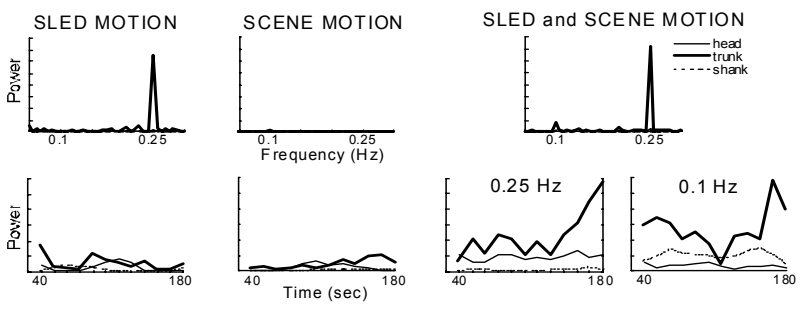

Fig. 4. Power plots of head, trunk, and shank in one LD adult plotted across frequency (top row) and over the period of the trial (bottom row) at the frequencies of sled motion only $(0.25 \mathrm{~Hz})$, scene motion only $(0.1 \mathrm{~Hz})$ and combined sled and scene motion. Responses are normalized to the largest response of each segment.

Response strategies were more consistent across the experimental conditions in the LD population. With sled motion only, the LD subject countered the stimulus with his head and trunk as a single unit and with little motion at the shank (Fig. 2). Scene motion alone generated very small segmental responses. When the inputs were combined, responses began to emerge at both frequencies in the trunk (Fig. 4). The head responded primarily to the support surface inputs and the shank exhibited increased power at the frequency of the scene. An increased reliance on visual signals has been documented in subjects with bilateral labyrinthine deficit [1,8], and it may be that in order to maintain an upright orientation this subject has learned to suppress or ignore discordant visual inputs. An increase in response magnitudes and power with combined inputs suggests these subjects could not completely suppress discordant stimuli when attention to multiple inputs was required.

\section{Principal Component Analysis}
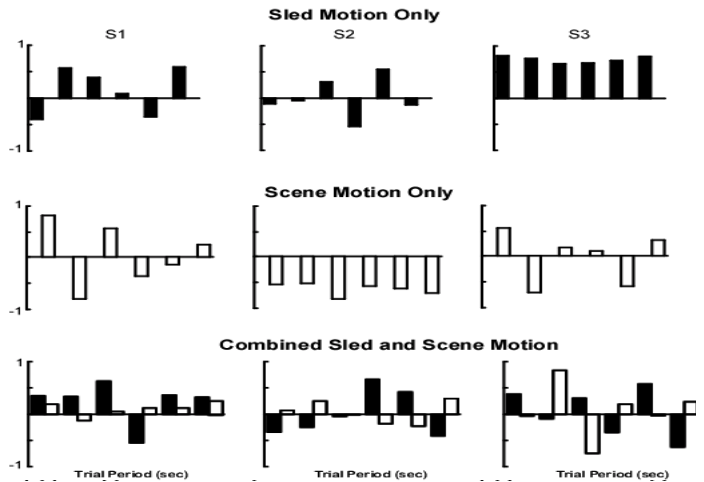

Fig. 5. Overall weighting of the input variables derived from the PCA for 3 young subjects. Each bar on a single axis represents a subsequent nonoverlapping $40 \mathrm{sec}$ time period. The direction of each bar indicates the relative phase between the response and the input signal. Responses to the platform are shown as filled bars; responses to the scene are shown as unfilled bars. 
We are also exploring the use of Principal Component Analysis (PCA) to determine the overall weighting of the input variables. In healthy young adults, some subjects consistently selected a single segmental strategy in the presence of a single input. With multiple inputs, most produced fluctuating behaviors. The relative weighting of each input fluctuated across a trial. Some subjects demonstrated consistent behaviors within a sensory set suggesting either proprioceptive (S3 in Fig. 5) or visual (S2 in Fig. 5) dominance. When the contribution of each body segment to the overall response strategy was calculated, movement was observed primarily in the trunk and shank.

\section{DISCUSSION}

The response to visual information was strongly potentiated by the presence of physical motion. Either stimulus alone produced marginal responses in most subjects. When combined, the response to visual stimulation was dramatically enhanced, perhaps because the visual inputs were incongruent with those of the physical motion. Even the LD subject responded to previously suppressed visual parameters when presented with multiple sensory demands. It is documented that individuals with bilateral labyrinthine deficit demonstrate gradually increasing instability in dynamic environments $[13,17,20]$. This would suggest that when in the presence of a multimodal, dynamic environment, vestibular information would be essential for resolving disparate inputs from the other sensory systems if postural responses were to be organized to compensate effectively for the multiple frequency disturbances [10]. It is possible that suppression of the continuous regulation of the visual environment results in a mismatch between their postural responses and environmental demands.

Current results and previous studies $[3,15]$ have shown that postural control has both time varying and frequency dependent properties. We found a non-additive effect in the energy of the response with combined inputs. With single inputs, some subjects consistently selected a single segmental strategy. With multiple inputs, most produced fluctuating behaviors. Individual perception of the sensory structure was a significant component of the postural response. Even in the absence of labyrinthine input, the combined effects of other sensory systems amplified the postural responses in a non-linear fashion and probably increased the potential for postural instability.

\section{CONCLUSION}

During functional activities, postural control is a continuous process and an integral component of any activity we engage in. Our current results demonstrate that postural control has both time varying and frequency dependent properties. Time dependent characteristics of postural responses might require a constant recalculation of the stimulus flow to appropriately tune the response to the environmental context. With labyrinthine deficit, an attempt to suppress components of the stimulus flow could produce a sensory mismatch that would interfere with the ability to compensate for environmental disturbances to balance. Thus, therapeutic interventions should require adaptation to complex, multimodal environments.

\section{REFERENCES}

[1] A.M. Bronstein, Visual vertigo syndrome: clinical and posturography findings. J. Neurol, Neurosurg, Psychiat., 59, 472-476, 1995.

[2] J. Buchanan and F. Horak, Emergence of postural patterns as a function of vision and translation frequency. J. Neurophysiol., 81, 2325-2339, 1999.

[3] J. P. Carroll and W. J. Freedman, Nonstationary properties of postural sway. J. Biomech., 26, 409-416, 1993.

[4] R. Creath, T. Kiemel, F. Horak, and J. J. Jeka, Limited control strategies with the loss of vestibular function. Exp. Brain Res., $145,323-333,2002$.

[5] J. Dichgans, R.Held, L.R. Young, and Th. Brandt, Moving visual scenes influence the apparent direction of gravity. Sci., 178, 1217-1219, 1972.

[6] J. Dichgans and Th. Brandt, "Visual-vestibular interactions and motion perception, in Handbook of Sensory Physiology. R. Held, H. W. Leibowitz, H-L Teuber, Eds. New York: Springer, 1978, vol. 8, pp. 755-804.

[7] H. Fushiki, S. Takata, and Y. Watanabe, Influence of fixation on circular vection. J. Vestib. Res., 10, 151-155, 2002.

[8] M. Guerraz, L. Yardley, P. Bertholon, L. Pollak, P. Rudge, M. A. Gresty, and A. M. Bronstein, Visual vertigo: symptom assessment, spatial orientation and postural control. Brain, 124, 1646-1656, 2001

[9] L. J. Hettinger, K. S. Berbaum, R. S. Kennedy, W. P. Dunlap, and M. D. Nolan, Vection and simulator sickness. Mil. Psychol., $2,171-181,1990$.

[10] I. P. Howard, Interactions within and between the spatial senses. J. Vestib. Res., 7, 311-347, 1997.

[11] E. A. Keshner and R. V. Kenyon, The influence of an immersive virtual environment on the segmental organization of postural stabilizing responses. J. Vestib. Res., 10, 201-219, 2000.

[12] E. A. Keshner, R. V. Kenyon, and J. Langston, Postural responses increase complexity with visual-vestibular discordance J. Vestib. Res., in press, 2004.

[13] E.A. Keshner, J.H.J. Allum, and C.R. Pfaltz, Postural coactivation and adaptation in the sway stabilizing responses of normals and patients with bilateral peripheral vestibular deficit. Exp. Brain Res., 69, 66-72, 1987.

[14] F. Lestienne, J. Soechting, and A. Berthoz, Postural readjustments induced by linear motion of visual scenes. Exp. Brain Res., 28, 363-384, 1977.

[15] P. J. Loughlin, M. S. Redfern, and J. M. Furman, Time-varying characteristics of visually induced postural sway. IEEE Trans. Rehab. Eng., 4, 416-424, 1996.

[16] K. S. Oie, T. Kiemel, and J. J. Jeka, Multisensory fusion: simultaneous re-weighting of vision and touch for the control of human posture. Cognit. Brain Res., 14, 164-176, 2002.

[17] N. G. R. Page and M. A. Gresty, Motorist's vestibular disorientation syndrome. J. Neurol. Neurosurg. Psychiat., 48, 729-735, 1985.

[18] R. J. Peterka, Sensorimotor integration in human postural control. J. Neurophysiol., 88, 1097-1118, 2002.

[19] T. Pozzo, A. Berthoz, and L. Lefort, Head kinematics during various motor tasks in humans. Prog. Brain Res., 80, 377-383, 1989.

[20] M. S. Redfern and J. M. Furman, Postural sway of patients with vestibular disorders during optic flow. J. Vestib. Res., 4, 221$230,1994$. 